\title{
Photodesorption of water ice
}

\section{A molecular dynamics study}

\author{
S. Andersson ${ }^{1,2,3}$ and E. F. van Dishoeck ${ }^{1}$ \\ 1 Leiden Observatory, Leiden University, PO Box 9513, 2300 RA Leiden, The Netherlands \\ e-mail: andersson@strw.leidenuniv.nl \\ 2 Gorlaeus Laboratories, Leiden Institute of Chemistry, Leiden University, PO Box 9502, 2300 RA Leiden, The Netherlands \\ Department of Chemistry, Physical Chemistry, Universtity of Gothenburg, 41296 Gothenburg, Sweden
}

Received 11 June 2008 / Accepted 24 September 2008

\section{ABSTRACT}

\begin{abstract}
Context. Absorption of ultraviolet radiation by water ice coating interstellar grains can lead to dissociation and desorption of the ice molecules. These processes are thought to be important in the gas-grain chemistry in molecular clouds and protoplanetary disks, but very few quantitative studies exist.

Aims. We compute the photodesorption efficiencies of amorphous water ice and elucidate the mechanisms by which desorption occurs.

Methods. Classical molecular dynamics calculations were performed for a compact amorphous ice surface at $10 \mathrm{~K}$ thought to be representative of interstellar ice. Dissociation and desorption of $\mathrm{H}_{2} \mathrm{O}$ molecules in the top six monolayers are considered following absorption into the first excited electronic state with photons in the 1300-1500 ̊̊range. The trajectories of the $\mathrm{H}$ and $\mathrm{OH}$ photofragments are followed until they escape or become trapped in the ice.

Results. The probability for $\mathrm{H}_{2} \mathrm{O}$ desorption per absorbed UV photon is $0.5-1 \%$ in the top three monolayers, then decreases to $0.03 \%$ in the next two monolayers, and is negligible deeper into the ice. The main $\mathrm{H}_{2} \mathrm{O}$ removal mechanism in the top two monolayers is through separate desorption of $\mathrm{H}$ and $\mathrm{OH}$ fragments. Removal of $\mathrm{H}_{2} \mathrm{O}$ molecules from the ice, either as $\mathrm{H}_{2} \mathrm{O}$ itself or its products, has a total probability of $2-3 \%$ per absorbed UV photon in the top two monolayers. In the third monolayer the probability is about $1 \%$ and deeper into the ice the probability of photodesorption falling to insignificant numbers. The probability of any removal of $\mathrm{H}_{2} \mathrm{O}$ per incident photon is estimated to be $3.7 \times 10^{-4}$, with the probability for photodesorption of intact $\mathrm{H}_{2} \mathrm{O}$ molecules being $1.4 \times 10^{-4}$ per incident photon. When no desorption occurs, the $\mathrm{H}$ and $\mathrm{OH}$ products can travel up to 70 and $60 \AA$ inside or on top of the surface, respectively, during which they can react with other species, such as $\mathrm{CO}$, before they become trapped.
\end{abstract}

Key words. astrochemistry - molecular data - ISM: molecules

\section{Introduction}

Ices are a major reservoir of the heavy elements in a variety of astrophysical environments, ranging from cold and dense molecular clouds (e.g., Willner et al. 1982; Whittet et al. 1988; Smith et al. 1989; Murakawa et al. 2000; Pontoppidan et al. 2004) and protoplanetary disks (Pontoppidan et al. 2005; Terada et al. 2007) to the icy bodies in our own solar system such as comets (e.g., Mumma et al. 1993) and Kuiper Belt Objects (Jewitt \& Luu 2004). In star-forming clouds, the fraction of carbon and oxygen locked up in ice is comparable to that in gaseous CO (Pontoppidan 2006; van Dishoeck et al. 1996), whereas at the centers of cold pre-stellar cores more than $90 \%$ of the heavy elements can be frozen out (e.g., Caselli et al. 1999; Bergin et al. 2002). Similarly, the cold midplanes of protoplanetary disks around young stars are largely devoid of gaseous molecules other than $\mathrm{H}_{2}, \mathrm{H}_{3}^{+}$and their isotopologues (e.g., Aikawa et al. 2002; Ceccarelli \& Dominik 2005). Thus, a good understanding of how molecules adsorb and desorb from the grains is critical to describe the chemistry in regions in which stars and planets are forming.

The importance of ultraviolet (UV) radiation in affecting interstellar ices is heavily debated in the literature. On the one hand, the large extinctions of $100 \mathrm{mag}$ or more along the lines of sight where ices are detected prevent UV radiation from penetrating deep into the clouds (e.g., Ehrenfreund et al. 2001; Stäuber et al. 2004), unless there are cavities through which the stellar UV photons can escape (Spaans et al. 1995). Thus, the bulk of the ices are thought to be shielded from both external and internal radiation sources in which case photodesorption is thought to be unimportant (e.g., Léger et al. 1985; Hartquist \& Williams 1990). On the other hand, UV photons are produced locally throughout the cloud by the interaction of cosmic rays with the gas, albeit at a level about $10^{4}$ times less than that of the general interstellar radiation field (Prasad \& Tarafdar 1983; Shen et al. 2004). In addition, X-rays from young stars penetrate much further into their surroundings than UV and can produce local UV photons through a similar process (Dalgarno et al. 1999; Stäuber et al. 2005). Moreover, the observed emission of optically thick millimeter lines from gaseous molecules is often dominated by the outer layers of the cloud where UV photons play a role. These UV photons can be important not only in the desorption of ices but also in the creation of reactive photoproducts such as energetic $\mathrm{H}$ atoms and radicals which can move through the ice and encounter other species leading to the formation of more complex molecules (e.g., d'Hendecourt et al. 1982; Garrod \& Herbst 2006).

Water ice is the dominant consituent of interstellar ices (e.g., Gibb et al. 2000; Pontoppidan 2006) with an abundance at least 
three orders of magnitude larger than that of gaseous water in cold clouds (Snell et al. 2000; Boonman et al. 2003). Thus, evaporation of water ice, even at a low fraction, can significantly affect the gaseous water abundance. Recent models of translucent and dense clouds invoke photodesorption of water ice in the outer regions to explain the gaseous water emission observed by the Submillimeter Wave Astronomy Satellite (SWAS) (Bergin $\&$ Melnick 2005). Photodesorption is also used to interpret the tentative detections of HDO and other gaseous species in the surface layers of protoplanetary disks (Willacy \& Langer 2000; Dominik et al. 2005). The adopted desorption efficiencies in these models, about $0.1 \%$ per incident UV photon, are based on a single experiment by Westley et al. (1995a,b) exposing ices at $35-100 \mathrm{~K}$ to Lyman- $\alpha$ radiation. The authors did not detect any water photodesorption in the limit of low UV photon fluence (integrated flux). Therefore it was suggested that water photodesorption only occurs from ices that have been subject to large doses of UV photons and not directly upon the first exposure to UV radiation. This is in contrast with recent experiments reported by Öberg et al. (2008), where there is a clear component of the water photodesorbed from amorphous ices at $18-100 \mathrm{~K}$ that is detected directly upon the first exposure to a UV lamp. Apart from that the results remain quite similar to the ones by Westley et al.

Other experiments on UV irradiation of water ices have also been performed. Ghormley \& Hochanadel (1971) observed H, $\mathrm{OH}$, and $\mathrm{H}_{2} \mathrm{O}_{2}$ following UV irradiation of crystalline ice at $263 \mathrm{~K}$, while Gerakines et al. (1996) found production of $\mathrm{OH}$, $\mathrm{HO}_{2}$, and $\mathrm{H}_{2} \mathrm{O}_{2}$ in the ice upon exposing amorphous ice at $10 \mathrm{~K}$ to UV light covering mainly the first and second electronic absorption bands of $\mathrm{H}_{2} \mathrm{O}$. Watanabe et al. (2000) irradiated amorphous $\mathrm{D}_{2} \mathrm{O}$ ice at $12 \mathrm{~K}$ with UV photons and observed substantial amounts of $\mathrm{D}_{2}$ after irradiation at $\lambda=126 \mathrm{~nm}$, but very little at $\lambda=172 \mathrm{~nm}$. In the experiments by Yabushita et al. (2006) $\mathrm{H}$ atoms were found to desorb from the ice after UV irradiation at $\lambda=157 \mathrm{~nm}$ and $\lambda=193 \mathrm{~nm}$. There are also a few reports on two- and multi-photon excitation of water ice leading to photodesorption of $\mathrm{H}_{2} \mathrm{O}$ molecules (Nishi et al. 1984; Bergeld \& Chakarov 2006). In these cases the photon energies are below the threshold for absorption in the ice, but upon multiple absorptions the excitation energies fall between 9 and $10 \mathrm{eV}$, between the first and second absorption band in ice. Clearly, there is a need for more quantitative information on the processes induced by UV photons in ices, even for the simplest cases such as pure water ice.

We present here the results of the first theoretical study of the dissociation of $\mathrm{H}_{2} \mathrm{O}$ molecules in pure water ice following absorption by UV photons. In addition to providing probabilities for desorption to be used in astrochemical models, these simulations provide insight into the mechanisms leading to desorption as well as the movements of the energetic photoproducts in the ice before they become trapped. In Sect. 2, we present the methods used in this study, in Sect. 3 the main results, and in Sect. 4 a short discussion and astrochemical implications. In Sect. 5 the results are summarized and some concluding remarks are given.

\section{Method}

All our calculations have been performed using classical Molecular Dynamics (MD) methods (Allen \& Tildesley 1987) with analytical potentials. Details of the computational procedure have been described in Andersson et al. (2006); here only a brief outline of the methods will be presented.

\subsection{Amorphous water ice}

To create an amorphous ice slab, the procedure outlined in Al-Halabi et al. (2004a) was used. In brief, a slab of 8 bilayers (16 monolayers) of crystalline ice was first created consisting of a cell containing $480 \mathrm{H}_{2} \mathrm{O}$ molecules. The cell has the dimensions $x$ : $22.4 \AA, y: 23.5 \AA$, and $z: 29.3 \AA$. Periodic boundary conditions are applied in the $x$ - and $y$-directions, the $z$ coordinate being parallel to the surface normal. Thus an infinite ice surface is created. The $\mathrm{H}_{2} \mathrm{O}$ molecules are treated as rigid rotors and their interactions are governed by the TIP4P potential (Jorgensen et al. 1983), which describes the interaction as a sum of pair interactions (electrostatic and Lennard-Jones potentials). The two bottom bilayers are kept fixed to simulate bulk ice and the molecules in the other 6 bilayers are allowed to move without any dynamical constraints other than that they remain rigid. The dynamics are at all times governed by classical Newtonian mechanics. To force the transition to amorphous ice, the surface is initially allowed to equilibrate for $5 \mathrm{ps}$ at $10 \mathrm{~K}$ but then the temperature is increased to $300 \mathrm{~K}$ using a computational equivalent of a thermostat (Berendsen et al. 1984). In this way the top bilayers form a liquid. The system is left to equilibrate for $100 \mathrm{ps}$ after which it is rapidly cooled to $10 \mathrm{~K}$. Then it is once again equilibrated for $100 \mathrm{ps}$. The resulting amorphous ice structure most closely resembles the structure of compact amorphous ice obtained experimentally and is thought to be representative of the structure of interstellar water ice (Al-Halabi et al. 2004a,b). See Fig. 4 of Al-Halabi et al. (2004a) and Fig. 2 of Andersson et al. (2006) for images. It does not exhibit the microporous structure that is obtained in vapor deposited ice (Mayer \& Pletzer 1986; Kimmel et al. 2001a,b). Given the dimensions of the simulation cell such a structure is simply not possible to obtain, since the pores should have a size on the same order as the cell we use. However, we believe that it is a good representation of an amorphous ice surface on a local scale, whether that be at the "outside" of the surface or inside a void deeper in the ice. Implications of this for the obtained results are discussed in Sect. 4.

In the rest of the paper we will discuss the depth into the ice in terms of monolayers. To avoid confusion our definition of monolayer is the thickness of ice corresponding to half a crystalline bilayer, i.e., if the ice were crystalline each bilayer would consist of two monolayers. For ease of definition the monolayers have been taken to be divided according to the $z$ values of the centers-of-mass of the molecules, e.g., the 30 molecules with the largest values of $z$ constitute the top ("first") monolayer.

\subsection{Initial conditions}

Once the ice surface is set up, one $\mathrm{H}_{2} \mathrm{O}$ molecule is chosen to be photodissociated. This molecule is then made completely flexible and its intramolecular (internal) interactions are governed by an analytic potential energy surface (PES) for the first electronically excited state (the $\widetilde{\mathrm{A}}^{1} \mathrm{~B}_{1}$ state) of gas-phase $\mathrm{H}_{2} \mathrm{O}$ based on high-quality ab initio electronic structure calculations (Dobbyn \& Knowles 1997). This excited potential is fully repulsive so that absorption into this state leads to dissociation of the $\mathrm{H}_{2} \mathrm{O}$ molecule into $\mathrm{H}+\mathrm{OH}$. The intermolecular interactions of the excited state $\mathrm{H}_{2} \mathrm{O}$ with the surrounding $\mathrm{H}_{2} \mathrm{O}$ molecules are governed by specially devised partial charges for describing the electrostatic interactions. In short, a charge of $-0.2 e$ is put on the $\mathrm{O}$ atom and charges of $+0.1 e$ on the $\mathrm{H}$ atoms. This gives a smaller dipole moment than that of the ground state $\mathrm{H}_{2} \mathrm{O}$ potential. The effect of this is that a less favorable interaction is 


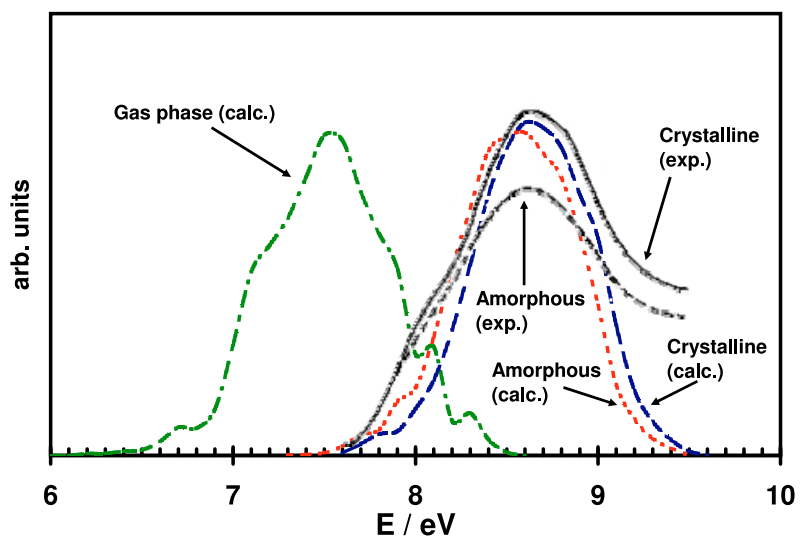

Fig. 1. Calculated and experimental (Kobayashi 1983) spectra of the first UV absorption band in crystalline and amorphous ices and calculated first absorption band for gas-phase $\mathrm{H}_{2} \mathrm{O}$. Note that the intensity is given in arbitrary units and that the intensities of the ice spectra have been scaled to roughly coincide.

obtained with the surrounding $\mathrm{H}_{2} \mathrm{O}$ molecules, giving higher excitation energies than with the uncorrected gas-phase potential energy surface. This leads to the blue-shift of about $1 \mathrm{eV}$ of the ice UV spectra seen in Fig. 1, which agree well with the first UV absorption band in amorphous and crystalline ices. If the ground state partial charges were to be used for the excited state the excitation spectrum would coincide with the gas-phase UV spectrum. For more details on the potentials see Andersson et al. (2006). Similar procedures using potential energy surfaces for higher excited states $(\tilde{\mathrm{B}}, \tilde{\mathrm{C}}$, etc.) should in principle give reasonable representations of higher-lying absorption bands in water ice.

The initial internal coordinates and momenta of the atoms in the selected molecule are sampled by a Monte Carlo procedure using a semi-classical (Wigner) phase-space distribution (Schinke 1993) that has been fitted to the ground-state vibrational wave function of $\mathrm{H}_{2} \mathrm{O}$ (van Harrevelt et al. 2001). This procedure gives initial conditions that are very similar to those found in fully quantum mechanical methods and has been shown to work well for the description of photodissociation processes of gaseous molecules. The transition dipole moment function, which governs the strength of the absorption, is taken from the calculation for gaseous $\mathrm{H}_{2} \mathrm{O}$ by van Harrevelt \& van Hemert (2000).

Dissociation of molecules in the top six monolayers has been considered. For each monolayer all 30 molecules have been dissociated, one molecule at a time. For each molecule 200 configurations and momenta were sampled from the Wigner distribution. This gives 6000 trajectories per monolayer and 36000 trajectories in total.

\subsection{Calculation of spectra}

The excitation energy is computed by taking the energy difference between an ice slab with an excited state $\mathrm{H}_{2} \mathrm{O}$ and one with a ground state $\mathrm{H}_{2} \mathrm{O}$ (with the same coordinates). Each excitation is assigned a weight calculated as the square of the coordinatedependent transition moment. By summing the weights of the excitation energies binned in $0.05 \mathrm{eV}$-wide energy intervals, "intensities" are obtained. Taken together these intensities form a UV absorption spectrum for the ice. The monolayers 5 to 6 were found to be converged to a "bulk behavior" (Andersson et al. 2005) and could therefore be used to compare the calculated spectra to experimental data. The gas-phase spectrum presented in Sect. 3 was obtained using the same intramolecular potential surfaces as above, but without the surrounding molecules and with 1000 sampled configurations.

\subsection{Dynamics of the dissociating molecule}

After putting the molecule in the excited state, the dissociating trajectory is integrated with a timestep of $0.02 \mathrm{fs}$. A maximum time of 20 ps has been used before terminating the trajectory. Most of the trajectories (99.6\%) were terminated before that because the system was found in one of the final outcomes (see Sect. 3) with negligible probability of transforming into a different state.

When the excited $\mathrm{H}_{2} \mathrm{O}$ dissociates, the intermolecular interactions are smoothly switched into separate interactions between the photoproducts and water ice, i.e., $\mathrm{H}-\mathrm{H}_{2} \mathrm{O}$ and $\mathrm{OH}-\mathrm{H}_{2} \mathrm{O}$ potentials. All details of the potentials and the functions used to switch between different potentials are given in Andersson et al. (2006). The switching functions connect the partial charges, the dispersion interactions and repulsive potentials between the $\mathrm{H}_{2} \mathrm{O}-\mathrm{H}_{2} \mathrm{O}$ potentials and the $\mathrm{OH}-\mathrm{H}_{2} \mathrm{O}$ and $\mathrm{H}-\mathrm{H}_{2} \mathrm{O}$ potentials affecting the dissociating molecule. The switches are functions of the $\mathrm{OH}$ distances $\left(R_{\mathrm{OH}}\right)$ within this molecule and will give the interaction parameters as continuous functions in the range 1.1-1.6 $\AA$ in $R_{\mathrm{OH}}$. The intramolecular potential is switched to the ground-state $\mathrm{PES}$, which allows $\mathrm{H}$ and $\mathrm{OH}$ to recombine to form $\mathrm{H}_{2} \mathrm{O}$. This switch is done in an analogous way as for the intermolecular interactions above, but here the range of $R_{\mathrm{OH}}$ where the switch is made is 3.0-3.5 $\AA$. In this range the excited-state and ground-state PES are near-degenerate, so a high transition probability between the two states is quite probable. Once $R_{\mathrm{OH}}$ becomes larger than $3.5 \AA$ the system will remain on the groundstate PES, even if $R_{\mathrm{OH}}$ again becomes smaller than $3.5 \AA$. This is what allows for recombination of $\mathrm{H}$ and $\mathrm{OH}$. The intermolecular interactions for the recombined ground state $\mathrm{H}_{2} \mathrm{O}$ with the surrounding $\mathrm{H}_{2} \mathrm{O}$ molecules are taken from the TIP3P potential (Jorgensen et al. 1983).

A slightly different stop criterion has been used in these calculations compared to the results presented previously (Andersson et al. 2005, 2006). When an $\mathrm{H}$ atom or $\mathrm{OH}$ is accommodated to the ice surface ("trapped") the trajectory is run until its translational energy equals $k_{\mathrm{B}} T$ or lower and the binding energy to the surface is $0.02 \mathrm{eV}$ ( $\mathrm{H}$ atom) or $0.1 \mathrm{eV}(\mathrm{OH})$ or stronger. In the older version of the code, the stop criterion was based on the kinetic energy of the individual atoms in relation to the potential energy (Andersson et al. 2006). The introduction of the new termination scheme led to a reduction by about $50 \%$ of the number of trajectories exceeding $20 \mathrm{ps}$.

Although most of the results presented here focus on amorphous ice, calculations have been performed for crystalline ice as well for comparison. Details can be found in Andersson et al. (2006).

\section{Results and discussion}

\subsection{Ice UV absorption spectrum}

As presented in Fig. 1, our calculated spectra of the first UV absorption bands in amorphous and crystalline ice match very well the experimentally obtained spectra, both in general shape as well as in the peak and threshold energies. The calculated gasphase spectrum of the first absorption band shown in Fig. 1 also 


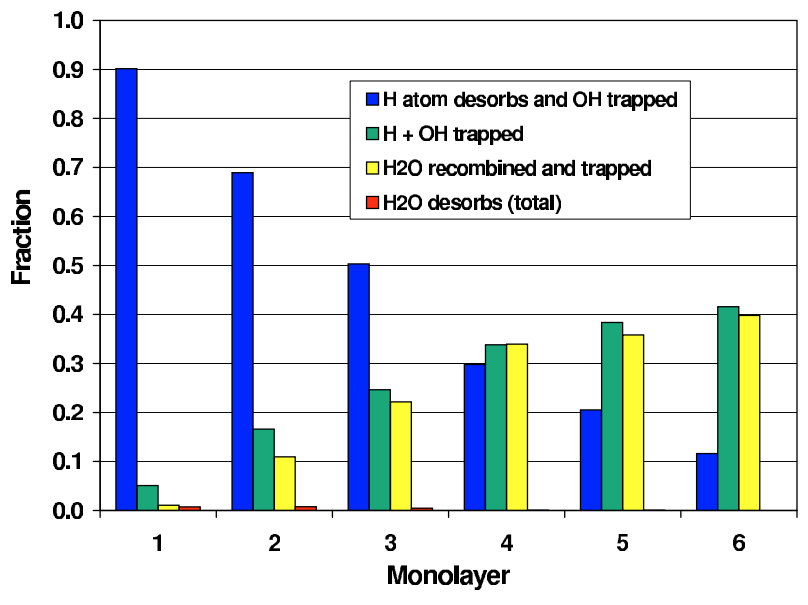

Fig. 2. Fractions of the main outcomes after $\mathrm{H}_{2} \mathrm{O}$ photodissociation for the top six monolayers of amorphous ice. These probabilities are calculated from all trajectories, irrespective of excitation energy.

matches the experimental peak energy (7.4-7.5 eV) quite well. This is naturally to be expected, since the potential surfaces used are based on very high-quality ab initio calculations of the energy. The success in reproducing the measured spectra leads us to believe that the amount of excess energy released into the ice is basically correct.

The ice spectrum is blue-shifted with respect to that of gaseous $\mathrm{H}_{2} \mathrm{O}$ and has significant cross section only in the 7.5-9.5 eV range. Thus, the photodesorption probabilities computed here are appropriate for photons in the 1300-1500 range. Dissociation of $\mathrm{H}_{2} \mathrm{O}$ can also occur following absorption into higher excited states (e.g., the equivalent of the $\tilde{\mathrm{B}}$ state of gaseous $\mathrm{H}_{2} \mathrm{O}$ ) but these generally contribute less than $20 \%$ of the total absorption in a dense cloud.

\subsection{Photoprocesses and desorption probabilities}

\subsubsection{Overall probabilities}

Photodissociation of a water ice molecule can have several outcomes, with the $\mathrm{H}$ and $\mathrm{OH}$ photoproducts either becoming trapped in the ice, recombining back to an $\mathrm{H}_{2} \mathrm{O}$ molecule, or desorbing from the ice surface. Figure 2 shows the probabilities (as fractions of the number of absorbed photons) of the main processes as functions of how deep into the ice the dissociating molecule initially is located. Note that these probabilities are given per absorbed UV photons and not per incident photon. In the first monolayer the dominant outcome is that the hydrogen atom desorbs and the $\mathrm{OH}$ radical is trapped in or on the ice. The probability of this event drops rapidly from about 0.9 in the first monolayer to just over 0.1 in the sixth monolayer. This reflects the effect of the ice on the motion of the $\mathrm{H}$ atom. At the surface there are very few molecules to stop the $\mathrm{H}$ atom from desorbing, but starting from deeper into the ice there are more obstacles on the way to the gas phase.

For the same reasons the probabilities of the other two major outcomes steadily increase as one moves deeper into the ice. These are the events when either both $\mathrm{H}$ and $\mathrm{OH}$ become separately trapped in the ice or when $\mathrm{H}$ and $\mathrm{OH}$ recombine to form $\mathrm{H}_{2} \mathrm{O}$ to subsequently become trapped in the ice. Except for the top two monolayers the probabilities of these two events are roughly equal and in the sixth monolayer the probabilities are up to 0.4 . The reason for the lower probability of the $\mathrm{H}_{2} \mathrm{O}$ molecule
Table 1. Total probabilities of $\mathrm{H}$ atom, $\mathrm{OH}$, and $\mathrm{H}_{2} \mathrm{O}$ desorption (per absorbed UV photon) as functions of monolayer.

\begin{tabular}{cccc}
\hline \hline ML & H atoms & OH & $\mathrm{H}_{2} \mathrm{O}$ \\
\hline 1 & 0.92 & 0.024 & $7.3 \times 10^{-3}$ \\
2 & 0.70 & 0.015 & $7.7 \times 10^{-3}$ \\
3 & 0.51 & $4.0 \times 10^{-3}$ & $4.8 \times 10^{-3}$ \\
4 & 0.30 & 0.00 & $3 \times 10^{-4}$ \\
5 & 0.21 & 0.00 & $3 \times 10^{-4}$ \\
6 & 0.12 & 0.00 & 0.00 \\
\hline
\end{tabular}

being recombined and trapped in the top monolayers can be understood from the open structure of the uppermost layers, which more easily allows for the photofragments to escape the region of the ice where they were initially formed.

The probability of photodesorption of $\mathrm{H}_{2} \mathrm{O}$ is seen to be low compared with the above processes, $0.7 \%$ in the top layer and $0.8 \%$ in the second layer, and then decreases with distance from the surface (see Table 1). However, this is only considering the desorption of intact $\mathrm{H}_{2} \mathrm{O}$ molecules. If one is interested in the removal of $\mathrm{H}_{2} \mathrm{O}$ from the surface without considering what enters the gas phase, the dominant mechanism in the top two monolayers is actually desorption of separate $\mathrm{H}$ and $\mathrm{OH}$ fragments. Desorption of $\mathrm{OH}$ is in most cases accompanied by the desorption of an $\mathrm{H}$ atom, but a minor fraction of $\mathrm{OH}$ desorption occurs with the $\mathrm{H}$ atom being trapped in the ice (see Sect. 3.2.2). In summary, the desorption probabilities of $\mathrm{OH}$ and $\mathrm{H}_{2} \mathrm{O}$ are about 2 orders of magnitude lower than that of $\mathrm{H}$ atoms with $\mathrm{OH}$ desorption being about twice as probable as $\mathrm{H}_{2} \mathrm{O}$ desorption if one sums over the probabilities from all monolayers (see also Sect. 4).

\subsubsection{Mechanisms and their probabilities}

Analysis of the trajectories shows that there are three distinct mechanisms for $\mathrm{H}_{2} \mathrm{O}$ removal (see Fig. 3). Note that these snapshots are taken from calculations on crystalline ice (Andersson et al. 2006) for ease of visualization: (a) An $\mathrm{H}$ atom released from photodissociation of $\mathrm{H}_{2} \mathrm{O}$ is able to transfer enough momentum to one of the other $\mathrm{H}_{2} \mathrm{O}$ molecules to "kick" it off the surface, (b) $\mathrm{H}$ and $\mathrm{OH}$ recombine to form $\mathrm{H}_{2} \mathrm{O}$ and subsequently desorb, and (c) the $\mathrm{H}$ and $\mathrm{OH}$ both desorb from the surface separately. In Table 2 the absolute probability of $\mathrm{H}_{2} \mathrm{O}$ desorption is given along with the relative probabilities of the three different mechanisms for each monolayer. With the additional mechanism (c) the probability of $\mathrm{H}_{2} \mathrm{O}$ removal is $2.7 \%$ and $1.9 \%$ per absorbed UV photon in the first and second monolayer, respectively.

In the top two monolayers the direct desorption of $\mathrm{H}$ and $\mathrm{OH}$ fragments is the dominant desorption mechanism, but in the third layer the three distinct desorption mechanisms ("a", "b", and "c") are roughly equally probable. Following UV absorption in monolayers 4 and 5 only the indirect "kick-out" mechanism is effective. In Fig. 4 the probabilities of all mechanisms of desorption of $\mathrm{H}$ atoms, $\mathrm{OH}$ radicals, and $\mathrm{H}_{2} \mathrm{O}$ molecules are presented for the top five monolayers. The desorption of $\mathrm{OH}$ is possible either together with the $\mathrm{H}$ atom as shown above or separately with the $\mathrm{H}$ atom remaining trapped. The former mechanism is found to have a higher probability. In total, the desorption of $\mathrm{OH}$ is about a factor of 2 more probable than the desorption of $\mathrm{H}_{2} \mathrm{O}$. Below the third monolayer the released $\mathrm{OH}$ radicals do not have sufficient kinetic energy to make it to the top of the surface and desorb. The rightmost three categories constitute a further 


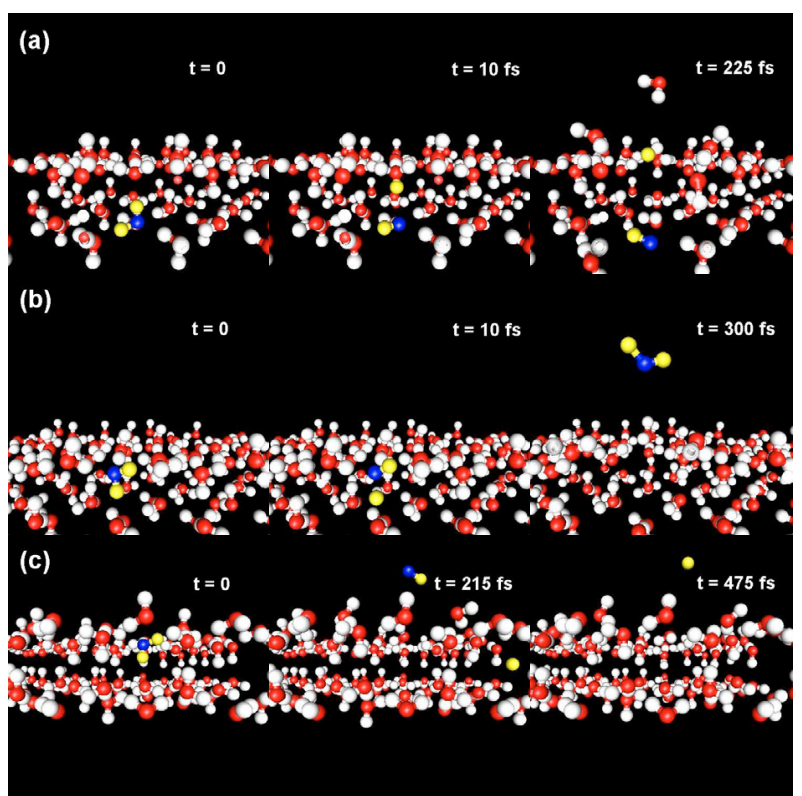

Fig. 3. Snapshots of trajectories of mechanisms of $\mathrm{H}_{2} \mathrm{O}$ desorption for a crystalline ice model. a) One of the surrounding molecules desorbs. b) The photofragments $\mathrm{H}$ and $\mathrm{OH}$ recombine and desorb as $\mathrm{H}_{2} \mathrm{O}$. c) The photofragments both desorb as separate species. The red and white atoms correspond to $\mathrm{O}$ and $\mathrm{H}$ atoms in the surrounding molecules and the blue and yellow atoms correspond to the $\mathrm{O}$ and $\mathrm{H}$ atoms of the photodissociated $\mathrm{H}_{2} \mathrm{O}$ molecule.

Table 2. Absolute probabilities for removal of $\mathrm{H}_{2} \mathrm{O}$ from an amorphous ice surface (per absorbed UV photon) as functions of monolayer.

\begin{tabular}{ccccc}
\hline \hline ML & $\begin{array}{c}\mathrm{H}_{2} \mathrm{O}^{a} \\
\text { desorption } \\
\text { probability }\end{array}$ & $\begin{array}{c}(\mathrm{a}) \\
\mathrm{H}_{2} \mathrm{O} \\
\text { intact }\end{array}$ & $\begin{array}{c}\left.\mathrm{H}_{2} \mathrm{O}\right) \\
\text { intact }\end{array}$ & $\begin{array}{c}\mathrm{H}+\mathrm{OH} \\
\text { fragments }\end{array}$ \\
\hline 1 & 0.027 & 0.10 & 0.17 & 0.73 \\
2 & 0.019 & 0.16 & 0.24 & 0.60 \\
3 & $7.0 \times 10^{-3}$ & 0.43 & 0.26 & 0.31 \\
4 & $3 \times 10^{-4}$ & 1.00 & 0.00 & 0.00 \\
5 & $3 \times 10^{-4}$ & 1.00 & 0.00 & 0.00 \\
\hline
\end{tabular}

${ }^{a}$ Probability for desorption as $\mathrm{H}_{2} \mathrm{O}$ or $\mathrm{H}+\mathrm{OH}$.

division of the category of indirect $\mathrm{H}_{2} \mathrm{O}$ desorption. Here " $\mathrm{H}_{2} \mathrm{O}$ indirect desorption" only refers to the cases where a molecule is kicked out by an $\mathrm{H}$ atom, which subsequently remains in the ice. The category " $\mathrm{H}_{2} \mathrm{O}$ desorption induced by recombination" refers to the rare occurence where it is the excess energy from the recombination of the $\mathrm{H}$ and $\mathrm{OH}$ fragments that kicks the molecule off the surface. The case " $\mathrm{H}+\mathrm{H}_{2} \mathrm{O}$ desorb" refers to when the $\mathrm{H}$ atom kicks the molecule off the surface and subsequently desorbs itself. This last category dominates the indirect desorption in the first two monolayers and also consitutes the maximum amount of matter that has been observed to desorb following photoexcitation. For $\mathrm{H}_{2} \mathrm{O}$ photoexcited below the fifth monolayer there is no evidence of photodesorption of $\mathrm{H}_{2} \mathrm{O}$ molecules.

The division into monolayers in Table 2 refers to where the photoexcited $\mathrm{H}_{2} \mathrm{O}$ is situated. The $\mathrm{H}_{2} \mathrm{O}$ molecules that actually desorb upon being expelled by an $\mathrm{H}$ atom or recombining $\mathrm{H}_{2} \mathrm{O}$ molecule all originate in monolayers 1 (84\%) and 2 (16\%). In most of these cases it is not only the transfer of momentum that is effective, but also the repulsive interaction from the photoexcited molecule, which most often is in the near vicinity of

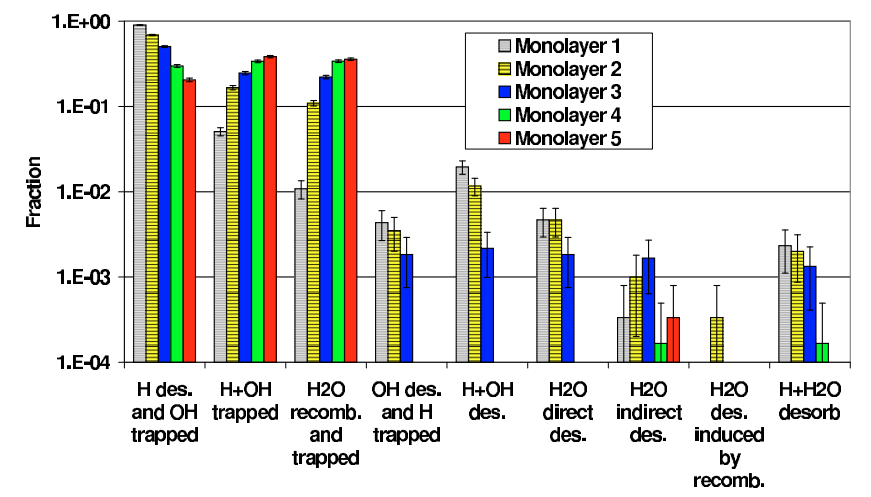

Fig. 4. Fractions of the detailed outcomes after $\mathrm{H}_{2} \mathrm{O}$ photodissociation for the top five monolayers of amorphous ice. The error bars correspond to a $95 \%$ confidence interval.

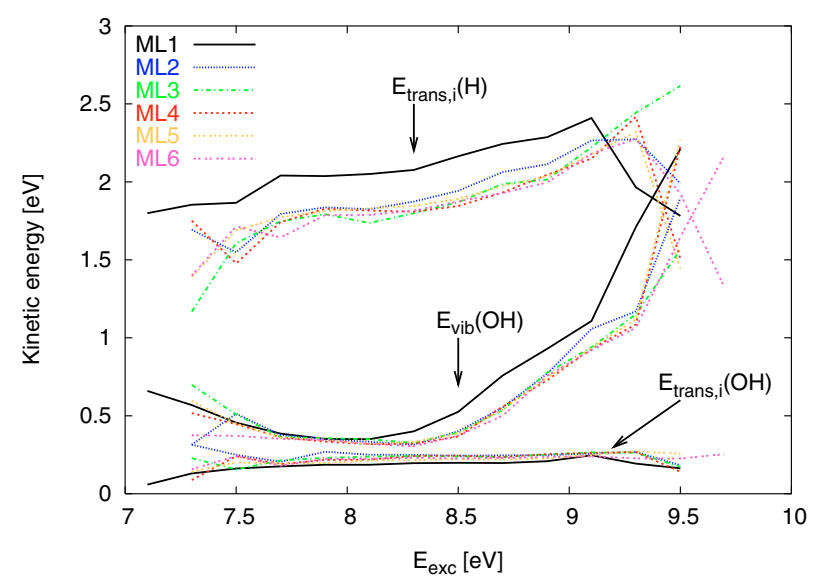

Fig. 5. The average initial translational energies of $\mathrm{H}$ atoms $\left[E_{\text {trans }, \mathrm{i}}(\mathrm{H})\right]$ and $\mathrm{OH}$ radicals $\left[E_{\text {trans, }}(\mathrm{OH})\right]$ and the average $\mathrm{OH}$ vibrational energy following photodissociation as function of excitation energy for the top six monolayers in amorphous ice.

the desorbing molecule. To illustrate this one can consider the lowering in binding energy of the molecule about to be desorbed. In monolayer 1 the average binding energy of all molecules is $0.9 \mathrm{eV}$ (calculated using the TIP4P potential) and of the desorbing molecules prior to excitation it is $0.8 \mathrm{eV}$. However, the photoexcitation lowers the binding energy of these molecules by on average $0.3 \mathrm{eV}$. About $25 \%$ of the desorbing molecules do not have their binding energy significantly lowered by excitation, but are kicked out solely by momentum transfer. If these molecules are excluded then the binding energy is on average lowered by $0.4 \mathrm{eV}$. In the second monolayer only about $10 \%$ of the desorbing molecules do not have their binding energy significantly lowered. There the average binding energy is $1.1 \mathrm{eV}$ and this is lowered by $0.3 \mathrm{eV}$ on average upon photoexcitation.

There is no sign of molecules being electronically excited and then desorbing intact directly, i.e., expelled by the repulsive interaction of the excited state molecule with its surroundings. The molecules dissociate very quickly (on the order of $10 \mathrm{fs}$ ) and that is not sufficient time for the molecule to desorb before it is dissociated. As discussed above the photofragments can however recombine and then subsequently desorb as $\mathrm{H}_{2} \mathrm{O}$.

\subsubsection{Effects of product energies}

The $\mathrm{H}$ atoms that are released have an average energy of 1.5-2.5 eV depending on the excitation energy and to a lesser 


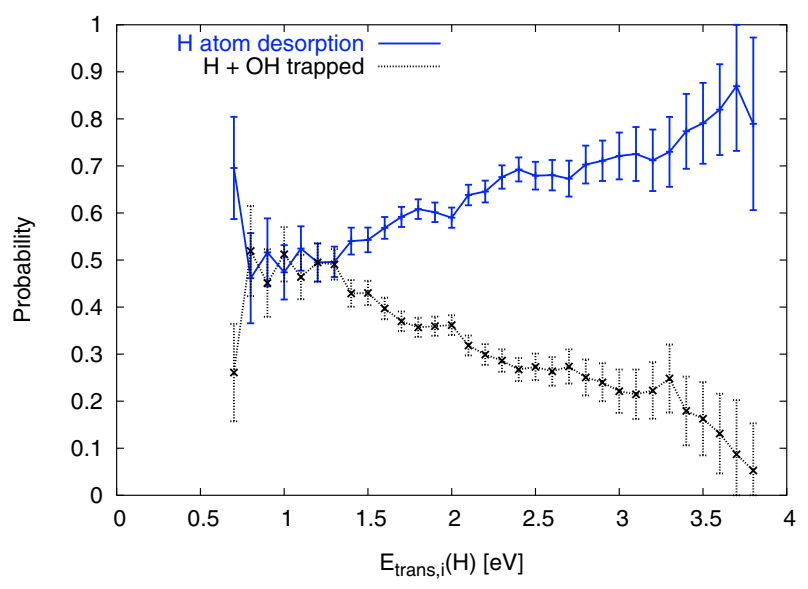

Fig. 6. The probabilities of $\mathrm{H}$ atom desorption and trapping of $\mathrm{H}$ and $\mathrm{OH}$ as function of initial $\mathrm{H}$ atom translational energy averaged over the top six monolayers in amorphous ice. Note that for technical reasons these are probabilities with the possibility of $\mathrm{H}_{2} \mathrm{O}$ recombination excluded (see text). The error bars correspond to a $95 \%$ confidence interval.

extent in which monolayer they originate (see Fig. 5). As can be seen the average initial $\mathrm{H}$ atom translational energy increases with increasing excitation energy, but above an excitation energy of $9 \mathrm{eV}$ it drops to somewhat lower energies. The average vibrational energy of $\mathrm{OH}$ has a minimum value of $0.3 \mathrm{eV}$ around $E_{\mathrm{exc}}=8 \mathrm{eV}$ but increases strongly to about $2 \mathrm{eV}$ at $E_{\text {exc }}=9.5 \mathrm{eV}$. This implies that at lower excitation energies the vast majority of the $\mathrm{OH}$ molecules are formed in the vibrational ground state, since the experimental zero-point energy of $\mathrm{OH}$ is $0.23 \mathrm{eV}$ (Huber \& Herzberg 1979) (see also Andersson et al. 2006). When the excitation energy is increased above $9 \mathrm{eV}$, large fractions of vibrationally excited $\mathrm{OH}$ are produced. The average initial translational energy of $\mathrm{OH}$ is only weakly dependent on excitation energy and lies around $0.2 \mathrm{eV}$ with only a slight increase with increasing $E_{\text {exc }}$.

In Fig. 6 the $\mathrm{H}$ atom desorption probability is plotted alongside the probability of $\mathrm{H}$ and $\mathrm{OH}$ both becoming trapped as functions of initial $\mathrm{H}$ atom translational energy. The plotted probabilities are somewhat higher than they should be because the probability of recombination of $\mathrm{H}_{2} \mathrm{O}$ has been excluded in the set of outcomes. The reason for this is that during recombination the translational energy of the $\mathrm{H}$ atom becomes very high. If recombination occurs immediately after dissociation it is quite difficult to distinguish the maximum translational energy the $\mathrm{H}$ atom normally would have after photodissociation and the maximum translational energy it gets during recombination. However, the trend is clear that the $\mathrm{H}$ atom desorption probability increases with increasing initial translational energy, as one would intuitively expect. The reason for the unexpectedly high desorption probability at $E_{\text {trans, } \mathrm{i}}(\mathrm{H})=0.7 \mathrm{eV}$ is not quite clear and it could simply be an effect of insufficient sample size, given that the error bars are fairly large.

Similarly, the desorption probability of $\mathrm{OH}$ has been plotted in Fig. 7 as function of initial $\mathrm{OH}$ translational energy in the top three monolayers. Also in this case the desorption probability increases with increasing initial kinetic energy. The effect is much stronger than for the case of $\mathrm{H}$ atoms, which reflects the much stronger binding energy of $\mathrm{OH}$ to its surroundings compared to that of the $\mathrm{H}$ atom. Interestingly, if the desorption probability is weighted with the initial distribution of translational energies, the total $\mathrm{OH}$ desorption probability (for the top three monolayers) increases rapidly at $0.2 \mathrm{eV}$ (the average initial translational

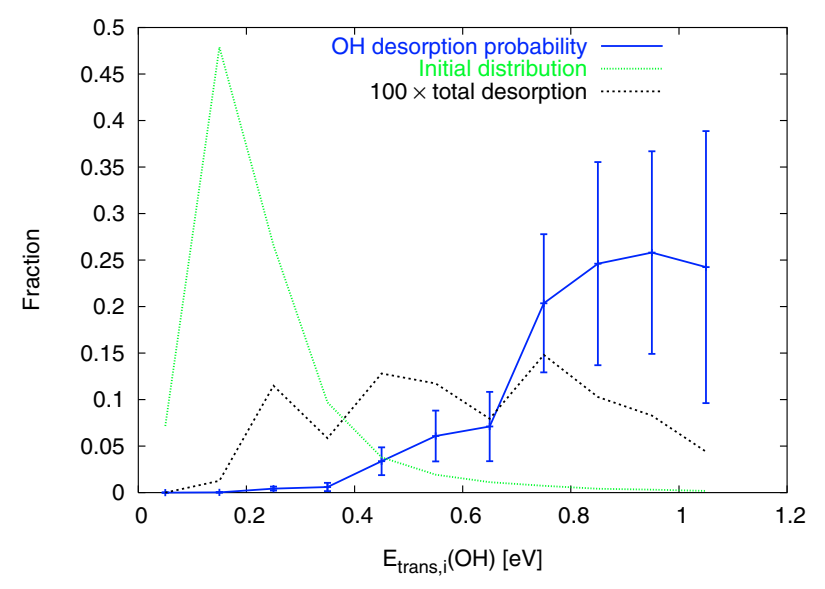

Fig. 7. The probabilities of $\mathrm{OH}$ desorption as functions of initial $\mathrm{OH}$ translational energy averaged over the three top monolayers of amorphous ice. Also shown are the initial distribution of $\mathrm{OH}$ translational energies and the total desorption probability (desorption probability $\times$ initial distribution). The error bars correspond to a $95 \%$ confidence interval.

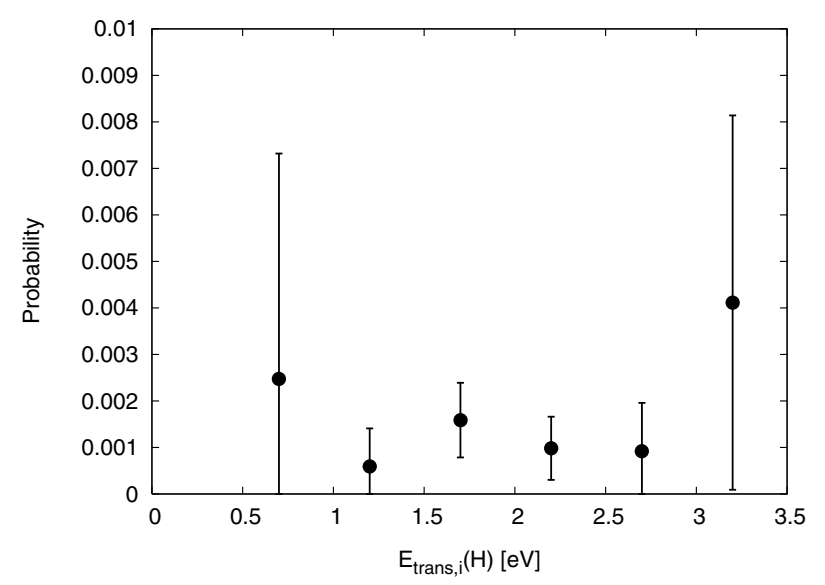

Fig. 8. Probability of indirect $\mathrm{H}_{2} \mathrm{O}$ desorption ("kick-out") as function of initial $\mathrm{H}$ atom translation energy (divided into bins of $0.5 \mathrm{eV}$ intervals) averaged over the top six monolayers of amorphous ice. The error bars correspond to a $95 \%$ confidence interval.

energy) and attains a basically constant value of $0.1 \%$ for all energies above that.

The dependence of the indirect $\mathrm{H}_{2} \mathrm{O}$ desorption probability on the translational energy of the $\mathrm{H}$ atom is found to be weak (see Fig. 8). There is not much evidence of any variation with translational energy and the desorption probability is fairly constant at $0.1 \%$ (averaged over the top six monolayers) over the whole energy range. It would be natural to expect that there could be a strong dependence on translational energy, but as discussed above the desorption is in most cases a combined effect of a repulsive force from the excited molecule, a lowered binding energy, and the momentum transfer from the $\mathrm{H}$ atom. Apparently, this allows also $\mathrm{H}$ atoms with relatively low translational energies to kick out $\mathrm{H}_{2} \mathrm{O}$ molecules.

\subsubsection{Dependence on photon energy}

Considering the desorption probabilities as functions of the excitation energy (Fig. 9) it is interesting to note that $\mathrm{H}$ atom desorption becomes less probable with increasing excitation energy ( 0.8 at $7.3 \mathrm{eV}$ and 0.3 at $9.5 \mathrm{eV}$ ). Since it was shown that the 


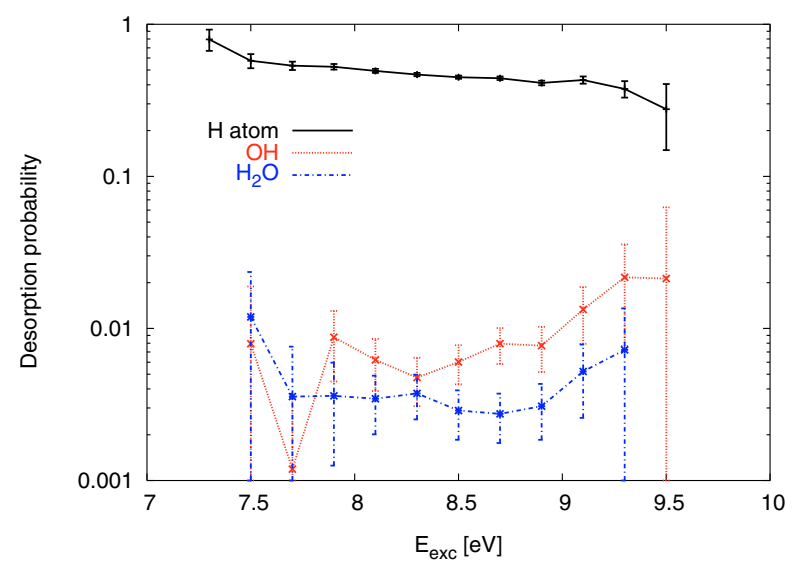

Fig. 9. Probabilities of desorption of $\mathrm{H}$ atoms, $\mathrm{OH}$ radicals, and $\mathrm{H}_{2} \mathrm{O}$ molecules as function of excitation energy averaged over the top six monolayers of amorphous ice. The error bars correspond to a $95 \%$ confidence interval.

average initial translational energy mainly increases with increasing excitation energy (Fig. 5) and that the desorption probability increases with increasing translational energy (Fig. 6) this seems like a paradox. The simple explanation of this behavior is that the lower excitation energies dominate in the top monolayers while the more energetic UV photons are mainly absorbed towards the bulk of the ice (see, e.g., Fig. 5 of Andersson et al. 2005). The desorption probability summed over the whole excitation energy range decreases rapidly with depth into the ice (Table 1) and therefore this unexpected behavior is found. The desorption of $\mathrm{OH}$ seems to increase with increasing excitation energy. This is a reflection of the fact that even though the $a v$ erage initial translational energy of $\mathrm{OH}$ varies only slightly over the excitation energy range (Fig. 5), there is a high-energy tail of the $\mathrm{OH}$ translational energy distribution (see Fig. 7) that becomes larger with higher excitation energies. The desorption probability of $\mathrm{H}_{2} \mathrm{O}$ does not show strong dependence on excitation energy, but considering the rather large error bars some energy dependence cannot be entirely ruled out.

\subsection{Mobility of photoproducts}

The $\mathrm{H}$ atoms produced in the photodissociation event are found to be quite mobile in the ice. On average the $\mathrm{H}$ atoms that become trapped move $8 \AA$ from their original locations. In extreme cases distances over $70 \AA$ are recorded. The $\mathrm{OH}$ radicals formed in the ice move only about $1 \AA$ with maximum distances moved of $5 \AA$. However, OH radicals formed from photodissociation in the top three monolayers are able in some cases to move tens of $\AA$ on top of the surface (up to more than $60 \AA$ ). The fact that some of the photofragments are able to move large distances implies an increased probability of reactions with other species in or on the ice, than if they would remain in the immediate vicinity of their point of origin. For more details see Andersson et al. (2006).

\subsection{Comparison to experiments}

In the experiments by Yabushita et al. (2006) on UV irradiation of polycrystalline and amorphous ices at $100 \mathrm{~K}$ absorption at $\lambda=157 \mathrm{~nm}\left(E_{\mathrm{exc}}=7.9 \mathrm{eV}\right)$ was found to result in $\mathrm{H}$ atom desorption. The translational energy distribution of the desorbing atoms was observed to consist of three components at $0.61 \mathrm{eV}$,

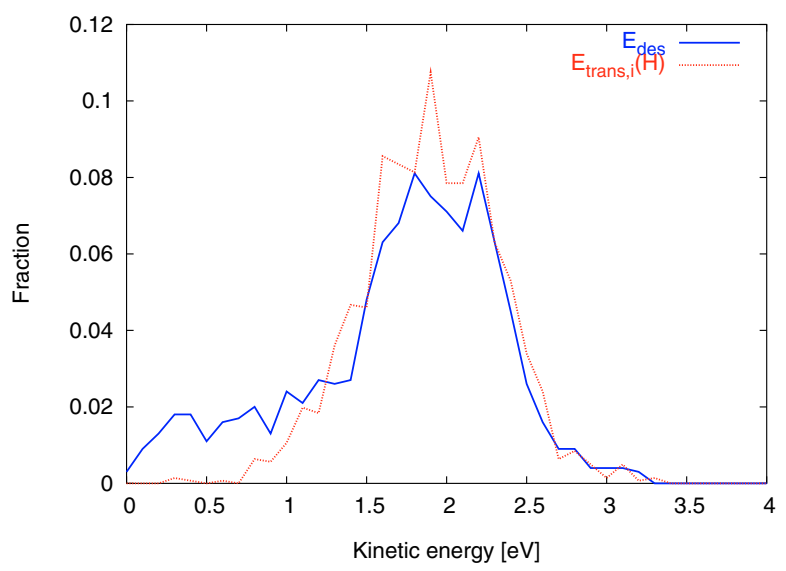

Fig. 10. Calculated distributions of $\mathrm{H}$ atom initial translational energies and desorption energies upon UV absorption at $E_{\mathrm{exc}}=7.9 \mathrm{eV}$ summed over the top six monolayers in amorphous ice.

$0.081 \mathrm{eV}$, and $0.014 \mathrm{eV}$, respectively. The lowest-energy component seems to consist of atoms that have thermalized prior to desorption. Thermal desorption occurs on a time scale that is likely much longer than would be feasible to do with molecular dynamics simulations. Therefore, it is not likely that we would see this third component in our calculations, but the other two should be possible to reproduce. In Fig. 10 the calculated distributions of initial translational energies and desorption energies of the released $\mathrm{H}$ atoms following excitation at $7.9 \mathrm{eV}$ are shown. It is seen that the initial translational energy has a peak at around $1.9 \mathrm{eV}$ and the desorption energy peaks at about the same energy. This clearly is much higher than found in the experiments, so it seems the energy of the desorbing $\mathrm{H}$ atoms is overestimated in our calculations. There could be three explanations to this behavior: (i) either the $\mathrm{H}$ atoms lose more energy prior to desorption or (ii) they are initially formed with less translational energy or (iii) a combination of these two effects. The possible sources of loss of highly energetic $\mathrm{H}$ atoms in the ice that cannot be treated by our calculations are: (a) the loss of energy by excitation of intramolecular modes in collisions with $\mathrm{H}_{2} \mathrm{O}$ molecules and (b) reactions with $\mathrm{H}_{2} \mathrm{O}$ molecules to form, e.g., $\mathrm{H}_{2}$ and $\mathrm{OH}$. For a thorough discussion see Andersson et al. (2006). Since we cannot directly tell how effective mechanism (i) is it is hard to speculate how much the desorption energy distribution is cooled through energy transfer and reaction. It is easier to speculate about mechanism (ii), since it is possible to monitor the dependence of the average desorption energy on the initial translational energy. If the initial translational energy is around $1 \mathrm{eV}$ the average desorption energies lie in the range $0.4-0.8 \mathrm{eV}$, which would be in much better accord with the experimentally measured desorption energies. If this is the most important mechanism for cooling the desorption energy distribution, then at $E_{\mathrm{exc}}=7.9 \mathrm{eV}$ the initial $\mathrm{H}$ atom translational energies are overestimated by roughly $1 \mathrm{eV}$. As discussed in Sect. 3.2 this would probably have little importance for the $\mathrm{H}_{2} \mathrm{O}$ desorption probability (Fig. 8). However, the desorption probability of $\mathrm{H}$ atoms could be somewhat lower than predicted in our calculations, but it is still quite likely to be of the same order of magnitude (Fig. 6). If the average $\mathrm{H}$ atom translational energy is overestimated it is also likely that the $\mathrm{OH}$ translational energy is somewhat overestimated. Considering the weak dependence on excitation energy (Fig. 5) the average $\mathrm{OH}$ translational energy might not be highly overestimated, but the high energy tail could be smaller, meaning that less $\mathrm{OH}$ radicals desorb than 
predicted here. If the initial kinetic energies of the photofragments are overestimated, the only possibility to account for the blue shift of the excitation energy is that the intermolecular repulsion is underestimated. This could have as an interesting effect that the indirect desorption of the surrounding molecules could be underestimated, since they would experience an even larger repulsive force from the excited molecule than predicted by our calculations. This remains to be investigated.

The high probabilities of $\mathrm{H}$ atom desorption is also in accordance with the finding of Gerakines et al. (1996) that their UVirradiated water ice was most likely depleted of $\mathrm{H}$ atoms, since a large amount of oxygen rich products was found. Our results on mobility of the released photofragments (Sect. 3.3, Andersson et al. 2006) have been supported by recent experiments by Elles et al. (2007), who observed an average separation of $\mathrm{H}$ and $\mathrm{OH}$ of $7 \pm 2 \AA$ immediately following photodissociation of $\mathrm{H}_{2} \mathrm{O}$ upon UV irradiation of liquid water. This is in excellent agreement with our calculated value of $8 \AA$ for the average distance from the site of photodissociation of the $\mathrm{H}$ atoms in amorphous ice. Even though the temperatures are quite different in the two cases, it is expected that liquid water and compact amorphous ice are quite similar on the short time scales during which photodissociation takes place.

A direct comparison with the water ice photodesorption results by Westley et al. (1995a,b) is difficult to make since they used Lyman- $\alpha$ radiation, which leads to excitation to a higher absorption band than considered here (Kobayashi 1983). Their findings of basically no photodesorption of intact $\mathrm{H}_{2} \mathrm{O}$ at low temperatures in the limit of single-photon absorption can therefore be neither refuted nor confirmed by our results. However, the detection of desorbing $\mathrm{H}_{2}$ and $\mathrm{O}_{2}$, and possibly $\mathrm{OH}$ and $\mathrm{H}_{2} \mathrm{O}_{2}$ actually agree with the present results (Westley et al. 1995a) (see below). In the experiments of Öberg et al. (2008) the photodesorbing material is detected in the form of $\mathrm{OH}, \mathrm{H}_{2} \mathrm{O}, \mathrm{H}_{2}$, and $\mathrm{O}_{2}$. This is the first time a positive detection of $\mathrm{OH}$ photodesorption has been reported. The detection of $\mathrm{OH}$ agrees nicely with our simulations (see also Sect. 4).

It is important to bear in mind that even though only $\mathrm{H}, \mathrm{OH}$, and $\mathrm{H}_{2} \mathrm{O}$ would desorb upon absorption of a single UV photon, in the experimental setup a large amount of UV photons may be absorbed in the ice during a relatively short time interval. This makes it possible to produce significant amounts of $\mathrm{H}$ and $\mathrm{OH}$ in the ice, and possibly also $\mathrm{O}$ atoms from for instance the photodissociation of the formed $\mathrm{OH}$ radicals. Given that thermal diffusion is effective in moving these reactive species into close contact, all the aforementioned desorbing species can be accounted for through recombination followed by desorption.

\section{Discussion and astrophysical implications}

Based on the results presented in this paper some important conclusions can be drawn on the possible outcomes of UV irradiation of water ice in interstellar environments. First, it is important to realize that by far the most likely species to desorb are $\mathrm{H}$ atoms, followed by $\mathrm{OH}$ radicals. In addition, when there is removal of $\mathrm{H}_{2} \mathrm{O}$ from the surface it seems likely that most of it comes off in the form of separate $\mathrm{H}$ and $\mathrm{OH}$ fragments. Therefore, there is not a one-to-one correspondence between $\mathrm{H}_{2} \mathrm{O}$ molecules removed from a surface and $\mathrm{H}_{2} \mathrm{O}$ appearing in the gas phase.

So far, this paper has been concerned with probabilities of photoinduced processes following absorption of one UV photon in a specific layer in the ice. However, not all incident UV photons are absorbed by molecules in the top six monolayers. To estimate desorption probabilities per incident photon rather than per absorbed photon one needs information about the absorption cross section. In our semiclassical simulations we are not able to calculate the absolute absorption cross section. However, Mason et al. (2006) have measured the absorption cross section of water ice at $25 \mathrm{~K}$ and found the peak absorption cross section in the first absorption band to be about $6 \times 10^{-18} \mathrm{~cm}^{2}$ at an excitation energy of $8.61 \mathrm{eV}$. This leads to an absorption probability of 0.007 photons $\mathrm{ML}^{-1}$ (see Appendix A for an outline of this calculation).

From the above estimate of the absorption probability it is possible to calculate photodesorption probabilities per incident UV photon. This is done by weighting the desorption probabilities per absorbed photon for each monolayer by the absorption probability for the specific monolayer with the absorption probabilities in any upper monolayers subtracted from the incoming photon flux. Using the information in Table 2 one arrives at a probability of removal of $\mathrm{H}_{2} \mathrm{O}$ from the ice of $3.7 \times 10^{-4}$ photon $^{-1}$. About $60 \%$ of the removed $\mathrm{H}_{2} \mathrm{O}$ comes off in the form of $\mathrm{H}+\mathrm{OH}, 20 \%$ desorbs as recombined $\mathrm{H}_{2} \mathrm{O}$, and the remaining $20 \%$ consists of $\mathrm{H}_{2} \mathrm{O}$ "kicked" out from the surface. The total photodesorption yield of intact $\mathrm{H}_{2} \mathrm{O}$ molecules is $1.4 \times 10^{-4}$ photon $^{-1}$. For $\mathrm{OH}$ desorption the probability is $3 \times 10^{-4}$ photon $^{-1}$, which includes both desorption with and without $\mathrm{H}$ atoms. The $\mathrm{H}$ atom photodesorption probability is relatively high, 0.02 photon $^{-1}$. The above ratios of photodesorption of $\mathrm{OH}$ and $\mathrm{H}_{2} \mathrm{O}$ are in excellent agreement with the recent experiments by Öberg et al. (submitted to ApJ), which inferred that roughly equal amounts of $\mathrm{OH}$ and $\mathrm{H}_{2} \mathrm{O}$ photodesorb at low surface temperature $(18 \mathrm{~K})$. They obtain a total photodesorption yield of about $1.3 \times 10^{-3}$ in the low-temperature limit, which is about 3 times higher than what is found from our simulations. Given the experimental uncertainties and the approximations made in the simulations this can be considered as good agreement. Commonly used estimates of $\mathrm{H}_{2} \mathrm{O}$ photodesorption probabilities in the range $1 \times 10^{-4}-3.5 \times 10^{-3}$ have been used to model different environments in agreement with observations (Bergin et al. 1995; Willacy \& Langer 2000; Snell et al. 2005; Dominik et al. 2005; Bergin \& Melnick 2005). Our results indicate that these estimates are reasonable. However, the finding that less than half of the desorbed material leaves the grain in the form of intact $\mathrm{H}_{2} \mathrm{O}$ molecules is something that should be included in the models.

The results presented here are only for photoinduced processes that are followed until the photoproducts desorb or are thermalized within the ice. This is all happening on a picosecond time scale. For longer time scales there is the possibility of thermal desorption of especially the $\mathrm{H}$ atoms. These are relatively weakly bound to the ice surface and it is quite probable that some fraction of the released $\mathrm{H}$ atoms desorb after thermalization in the ice. This contribution to the desorption probability is therefore not included in the above estimate.

It is interesting to discuss the possible effects of the overall morphology of the ice surface. It is clear that the vast majority of water ice surfaces in the interstellar medium are amorphous (Hagen et al. 1981). However, it is debated whether the ice is mostly porous, as found in vapor deposited ice (Mayer \& Pletzer 1986; Kimmel et al. 2001a,b), or more compact (Fraser et al. 2004; Palumbo 2006). A complicating factor is that most likely the ice is formed through chemical reactions on grains rather than accretion of $\mathrm{H}_{2} \mathrm{O}$ molecules from the gas phase (O'Neill \& Williams 1999). Therefore, the exact ice morphology that results from such a chemical build-up of the ice is not yet clear (see however Cuppen \& Herbst 2007). If a porous ice surface is 
subjected to UV irradiation one could have release of $\mathrm{H}, \mathrm{OH}$, or $\mathrm{H}_{2} \mathrm{O}$ into a void in the ice rather than directly into the gas phase. This is inferred to happen in the experiments by Yabushita et al. (2006) where a large component of the desorbing $\mathrm{H}$ atoms released after UV irradiaton of amorphous ice are thermalized, likely due to $\mathrm{H}$ atoms being accommodated within a void in the ice and then desorbing thermally. Some $\mathrm{OH}$ and $\mathrm{H}_{2} \mathrm{O}$ could photodesorb in a similar way. However, if the path to reach the gas phase from inside a pore is restricted, these species have a high probability of being trapped inside a pore because of their strong attractive interactions with the ice. In practice, the $\mathrm{OH}$ and $\mathrm{H}_{2} \mathrm{O}$ released in this way might not show up in desorption. Therefore, if a porous ice is considered it is necessary to distinguish between the surface area that is directly exposed to the gas phase and that which is within a void with restricted access to the outside. In conclusion, the $\mathrm{H}$ atom desorption from a porous ice is likely to be different from that of compact nonporous ice, but the desorption yields of $\mathrm{OH}$ and $\mathrm{H}_{2} \mathrm{O}$ are not necessarily very different in the two types of ice.

The cosmic ray induced UV flux inside dense clouds is about $10^{4} \mathrm{~cm}^{-2} \mathrm{~s}^{-1}$ (Shen et al. 2004). For an ice-coated grain with a typical size of $0.1 \mu \mathrm{m}$, this would give an arrival rate of about $1 \mathrm{UV}$ photon per day. The case of a single UV absorption event as described in our simulations therefore gives a realistic picture of photodesorption in dense clouds. In the laboratory, the UV flux is many orders of magnitude higher and multiple absorptions of UV photons within the ice surface in a short time interval may drive secondary reactions of photodissociation products from different $\mathrm{H}_{2} \mathrm{O}$ molecules. Experiments with different UV flux levels down to low levels will be needed to provide quantitative data on photodesorption yields relevant for astrophysical applications.

As has also been noted in our previous work another important aspect is the release of reactive species into the ice. That would have implications for reactivity in the ice with, e.g., $\mathrm{CO}$ that could react with energetic $\mathrm{H}$ or $\mathrm{OH}$ to form $\mathrm{HCO}$ or $\mathrm{CO}_{2}$. This could be one clue to unraveling the mystery of $\mathrm{CH}_{3} \mathrm{OH}$ and $\mathrm{CO}_{2}$ formation in the interstellar medium. Indeed, formation of $\mathrm{CO}_{2}$ is readily observed when a mixed $\mathrm{H}_{2} \mathrm{O}: \mathrm{CO}$ ice is photolysed (d'Hendecourt et al. 1986; Watanabe \& Kouchi 2002; Watanabe et al. 2007).

\section{Conclusions}

We have shown that it is possible to have $\mathrm{H}_{2} \mathrm{O}$ photodesorption upon UV absorption to the first absorption band in the top five monolayers of an amorphous ice surface. The main mechanisms for this photodesorption are either photodissociation followed by recombination of $\mathrm{H}$ and $\mathrm{OH}$ and subsequent desorption of the recombined $\mathrm{H}_{2} \mathrm{O}$ molecule or a "kick-out" of another $\mathrm{H}_{2} \mathrm{O}$ molecule in the ice by the energetic $\mathrm{H}$ atom released from photodissociation or, less likely, by the energy released from a recombined $\mathrm{H}_{2} \mathrm{O}$ molecule. In most cases, however, removal of an $\mathrm{H}_{2} \mathrm{O}$ molecule from the ice is in the form of separate $\mathrm{H}$ and $\mathrm{OH}$ fragments. An estimate of the photodesorption yield per incident UV photon from our calculations agrees well with the $\mathrm{H}_{2} \mathrm{O}$ photodesorption yields that are commonly used in modeling astrophysical environments.

$\mathrm{UV}$ absorption leads in most cases to desorption of $\mathrm{H}$ atoms or the trapping of $\mathrm{H}$ and $\mathrm{OH}$ in the ice either as separate fragments or as recombined $\mathrm{H}_{2} \mathrm{O}$. The desorption of $\mathrm{H}$ atoms is about 2 or 3 orders of magnitude more probable than desorption of $\mathrm{OH}$ and $\mathrm{H}_{2} \mathrm{O}$. The $\mathrm{OH}$ desorption probability is about twice the $\mathrm{H}_{2} \mathrm{O}$ desorption probability. The high mobility of $\mathrm{H}$ atoms inside the ice and $\mathrm{OH}$ radicals on the ice surface will facilitate formation of other molecules such as $\mathrm{CO}_{2}$.

Acknowledgements. We thank Karin Öberg, Herma Cuppen, and Geert-Jan Kroes for stimulating discussions. Some of the calculations reported here were performed at Chalmers Centre for Computational Science and Engineering (C3SE) computing resources. This research was funded by a Netherlands Organization for Scientific Research (NWO) Spinoza grant (for one of the authors (E.F.v.D)) and a NWO-CW Top grant.

\section{Appendix A: Estimate of absorption probability}

To calculate an absorption probability per monolayer in an interstellar ice surface one needs an estimate of the effective area taken up by one molecule. Since the angle of incidence of the photon is arbitrary, all possible incidence angles have to be taken into account, not only normal incidence. The surface area of our simulation cell is $22.4 \AA \times 23.5 \AA=526 \AA^{2}=5.26 \times 10^{-14} \mathrm{~cm}^{2}$. If one assumes a flat surface, the average effective surface area seen by a photon from any incidence angle between $0^{\circ}$ (normal incidence) and $90^{\circ}$ (parallel to the surface) is the actual surface area divided by two. This is arrived upon by the following expression:

$\left\langle A_{\mathrm{eff}}\right\rangle=\frac{\int_{0}^{\pi / 2} A \cos \theta \sin \theta \mathrm{d} \theta}{\int_{0}^{\pi / 2} \sin \theta \mathrm{d} \theta}=\frac{A}{2}$,

with the effective area given by $A_{\text {eff }}=A \cos \theta$ where $A$ is the actual surface area and $\theta$ is the angle of incidence. The average effective surface area of the simulation cell is $2.63 \times$ $10^{-14} \mathrm{~cm}^{2}$. Each monolayer consists of $30 \mathrm{H}_{2} \mathrm{O}$ molecules. A single molecule will therefore have an average effective area $\left\langle A_{\text {eff }}^{\text {mol }}\right\rangle=8.77 \times 10^{-16} \mathrm{~cm}^{2}$. Mason et al. (2006) measured the peak absorption cross section, $\sigma$, in the first absorption band to be about $6 \times 10^{-18} \mathrm{~cm}^{2}$ around an excitation energy of $8.61 \mathrm{eV}$. With this value the absorption probability per monolayer becomes $P_{\mathrm{abs}}^{\mathrm{ML}}=\sigma /\left\langle A_{\mathrm{eff}}^{\mathrm{mol}}\right\rangle=7 \times 10^{-3}$ (this number may vary somewhat with excitation energy). If one considers an infinitely deep ice surface all photons will be absorbed within the ice. For an ice surface in the interstellar medium consisting of a finite number of layers, the value of $P_{\mathrm{abs}}^{\mathrm{ML}}$ may vary depending on the shape of the grain, since the surface is not necessarily flat. However, it is likely that any photon that passes through an ice mantle will be absorbed by the silicate grain, leading to almost total absorption of the incident photons within the grain.

\section{References}

Aikawa, Y., van Zadelhoff, G. J., van Dishoeck, E. F., \& Herbst, E. 2002, A\&A, 386,622

Al-Halabi, A., van Dishoeck, E. F., \& Kroes, G. J. 2004a, J. Chem. Phys., 120, 3358

Al-Halabi, A., Fraser, H. F., Kroes, G. J., \& van Dishoeck, E. F. 2004b, A\&A, 422, 777

Allen, M. P., \& Tildesley, D. J. 1987, Computer Simulations of Liquids (Oxford: Clarendon)

Andersson, S., Kroes, G. J., \& van Dishoeck, E. F. 2005, Chem. Phys. Lett., 408, 415

Andersson, S., Al-Halabi, A., Kroes, G. J., \& van Dishoeck, E. F. 2006, J. Chem. Phys., 124, 064715

Berendsen, H. J. C., Postma, J. P. M., van Gunsteren, W. F., DiNola, A., \& Haak, J. R. 1984, J. Chem. Phys., 81, 3684

Bergeld, J., \& Chakarov, D. 2006, J. Chem. Phys., 125, 141103

Bergin, E. A., \& Melnick, G. 2005, in Astrochemistry: recent successes and current challenges, ed. D. C. Lis, G. A. Blake, \& E. Herbst (Cambridge: Cambridge University Press), IAU Symp., 231, 309

Bergin, E. A., Langer, W. D., \& Goldsmith, P. F. 1995, ApJ, 441, 222

Bergin, E. A., Alves, J., Huard, T., \& Lada, C. J. 2002, ApJ, 570, L101 
Boonman, A. M. S., Doty, S. D., van Dishoeck, E. F., et al. 2003, A\&A, 406, 937 Caselli, P., Walmsley, C. M., Tafalla, M., Dore, L., \& Myers, P. C. 1999, ApJ, 523, L165

Ceccarelli, C., \& Dominik, C. 2005, A\&A, 440, 583

Cuppen, H. M., \& Herbst, E. 2007, ApJ, 668, 294

Dalgarno, A., Yan, M., \& Liu, W. 1999, ApJS, 125, 237

d'Hendecourt, L. B., Allamandola, L. J., Baas, F., \& Greenberg, J. M. 1982, A\&A, 109, L12

d'Hendecourt, L. B., Allamandola, L. J., Grim, R. J. A., \& Greenberg, J. M. 1986, A\&A, 158, 119

Dobbyn, A. J., \& Knowles, P. J. 1997, Mol. Phys., 91, 1107

Dominik, C., Ceccarelli, C., Hollenbach, D., \& Kaufman, M. 2005, ApJ, 635, L85

Ehrenfreund, P., d'Hendecourt, L., Charnley, S., \& Ruiterkamp, R. 2001, J. Geophys. Res., 106, 33291

Elles, C. G., Shkrob, I. A., Crowell, R. A., \& Bradforth, S. E. 2007, J. Chem. Phys., 126, 164503

Fraser, H. J., Collings, M. P., Dever, J. W., \& McCoustra, M. R. S. 2004, MNRAS, 353, 59

Garrod, R. T., \& Herbst, E. 2006, A\&A, 457, 927

Gerakines, P. A., Schutte, W. A., \& Ehrenfreund, P. 1996, A\&A, 312, 289

Ghormley, J. A., \& Hochanadel, C. J. 1971, J. Phys. Chem., 75, 40

Gibb, E. L., Whittet, D. C. B., Schutte, W. A., et al. 2000, ApJ, 536, 347

Hagen, W., Tielens, A. G. G. M., \& Greenberg, J. M. 1981, Chem. Phys., 56, 367

Hartquist, T. W., \& Williams, D. A. 1990, MNRAS, 247, 343

Huber, K. P., \& Herzberg, G. 1979, Molecular Spectra and Molecular Structure, IV, Constants of Diatomic Molecules (New York: Van Nostrand)

Jewitt, D. C., \& Luu, J. 2004, Nature, 432, 731

Jorgensen, W. L., Chandrasekhar, J., Madura, J. D., Impey, R. W., \& Klein, M. L. 1983, J. Chem. Phys., 79, 926

Kimmel, G. A., Stevenson, K. P., Dohnálek, Z., Scott Smith, R., \& Kay, B. D. 2001a, J. Chem. Phys., 114, 5284

Kimmel, G. A., Dohnálek, Z., Stevenson, K. P., Scott Smith, R., \& Kay, B. D. 2001b, J. Chem. Phys., 114, 5295

Kobayashi, K. 1983, J. Phys. Chem., 87, 4317

Léger, A., Jura, M., \& Omont, A. 1985, A\&A, 144, 147

Mason, N. J., Dawes, A, Holtom, P. D., et al. 2006, Faraday Discuss., 133, 311

Mayer, E., \& Pletzer, R. 1986, Nature, 319, 298

Mumma, M. J., Weissman, P. R., \& Stern, S. A. 1993, in Protostars \& Planets III, ed. E. H. Levy, \& J. I. Lunine, Tucson, University of Arizona, 1177
Murakawa, K., Tamura, M., \& Nagata, T. 2000, ApJS, 128, 603

Nishi, N., Shinohara, H., \& Okuyama, T. 1984, J. Chem. Phys., 80, 3898

O’Neill, P. T., \& Williams, D. A. 1999, Ap\&SS, 266, 539

Öberg, K. I., Visser, R., van Dishoeck, E. F., \& Linnartz, H. 2008, ApJ, submitted Palumbo, M. E. 2006, A\&A, 453, 903

Pontoppidan, K. M. 2006, A\&A, 453, L47

Pontoppidan, K. M., van Dishoeck, E. F., \& Dartois, E. 2004, A\&A, 426, 925

Pontoppidan, K. M., Dullemond, C. P., van Dishoeck, E. F., et al. 2005, ApJ, 622, 463

Prasad, S. S., \& Tarafdar, S. P. 1983, ApJ, 267, 603

Schinke, R. 1993, Photodissociation Dynamics (Cambridge: Cambridge University Press)

Shen, C., Greenberg, J. M., Schutte, W. A., \& van Dishoeck, E. F. 2004, A\&A, 415, 203

Smith, R. G., Sellgren, K., \& Tokunaga, A. T. 1989, ApJ, 344, 413

Snell, R. L., Howe, J. E., Ashby, M. L. N., et al. 2000, ApJ, 539, L97

Snell, R. L., Hollenbach, D., Howe, J. E., et al. 2005, ApJ, 620, 758

Spaans, M., Hogerheijde, M. R., Mundy, L. G., \& van Dishoeck, E. F. 1995, ApJ, 455, L167

Stäuber, P., Doty, S. D., van Dishoeck, E. F., Jørgensen, J. K., \& Benz, A. O. 2004, A\&A, 425, 577

Stäuber, P., Doty, S. D., van Dishoeck, E. F., \& Benz, A. O. 2005, A\&A, 440, 949

Terada, H., Tokunaga, A. T., Kobayashi, et al. 2007, ApJ, 667, 303

van Dishoeck, E. F., Helmich, F. P., de Graauw, T., et al. 1996, A\&A, 315, L349

van Harrevelt, R., \& van Hemert, M. C. 2000, J. Chem. Phys., 112, 5777

van Harrevelt, R., van Hemert, M. C. \& Schatz, G. C. 2001, J. Phys. Chem. A, 105,11480

Watanabe, N., \& Kouchi, A. 2002, ApJ, 567, 651

Watanabe, N., Horii, T., \& Kouchi, A. 2000, ApJ, 541, 772

Watanabe, N., Mouri, O., Nagaoka, A., et al. 2007, ApJ, 668, 1001

Westley, M. S., Baragiola, R. A., Johnson, R. E., \& Baratta, G. A. 1995a, Planet. Space Sci., 43, 1311

Westley, M. S., Baragiola, R. A., Johnson, R. E., \& Baratta, G. A. 1995b, Nature, 373, 405

Whittet, D. C. B., Bode, M. F., Longmore, A. J., et al. 1988, MNRAS, 233, 321

Willacy, K., \& Langer, W. D. 2000, ApJ, 544, 903

Willner, S. P., Gillett, F. C., Herter, T. L., et al. 1982, ApJ, 253, 174

Yabushita, A., Kanda, D., Kawanaka, N., Kawasaki, M., \& Ashfold, M. N. R. 2006, J. Chem. Phys., 125, 133406 